DOE/ER/40561-298-INT96-21-003

\title{
Frame Dependence of Parton Cascade results
}

CU-TP-794

\author{
Bin Zhang \\ Department of Physics, Columbia University, New York, NY 10027 \\ and \\ INT, University of Washington, Seatle, WA 98195-1550 \\ Yang Pang \\ Department of Physics, Columbia University, New York, NY 10027
}

(6th November 1996)

\begin{abstract}
Frame dependence of parton cascade results is studied for different schemes of doing cascade simulations. We show that different schemes do not always agree and results may have strong frame dependence. When the interaction range is on the order of mean free path and the collisions are done in the two parton center of mass frame, the results are not sensitive to the global frame that the collisions are ordered. Effects of different differential cross sections are also discussed.
\end{abstract}

\section{INTRODUCTION}

Cascade models have been widely used to simulate Relativistic and Ultra-Relativistic Nucleus-Nucleus collisions [1]. They can be used to simulate systems that are not in thermal equilibrium and they incooperate the effect of finite mean free path automatically.

Causality violation is inherent in cascade simulations due to the geometrical interpretation of the cross section, i.e., particles scatter when their closest distance is smaller than $\sqrt{\frac{\sigma}{\pi}}$ ( $\sigma$ is the scattering cross section). One problem is that the information travels across $\sqrt{\frac{\sigma}{\pi}}$ at a time which is either the fixed time step or the mean free path. This may lead to a speed of information faster than the speed of light and may cause superluminous shock waves. Another problem is that different choices of doing collisions, i.e., different collision schemes, may lead to different collision orderings and hence different physical predictions.

Many groups have studied the causality problem for energies below RHIC energy [2] and attempts have been made to reduce the problem. For Ultra-relativistic heavy ion collisions at RHIC energies and beyond, causality violation may be very serious due to the relatively small mean free path which may be on the order or even much less than the interaction range. The superluminous signals have been studied [3] in cascade simulations of $\mathrm{pA}$ collisions. In this paper, we study the effect of collision ordering on macroscopic variables by comparing the results of different collision schemes. A parton cascade code, GPC (Generic Parton Cascade), we developed recently [4] has been used in this study.

First, we describe the initial conditions and different collision schemes. Then we compare the results from different schemes with a discussion of the effects of different differential cross sections followed by the conclusions.

\section{INITIAL CONDITIONS AND COLLISION SCHEMES}

To study frame dependence of cascade results at RHIC energies, we prepare a system of gluons similar to the minijet gluon system that is going to be produced at RHIC. 4000 gluons are uniformly distributed in the -5 to 5 space time rapidity range. Initially, they get local thermal equilibrium at temperature $500 \mathrm{MeV}$. They occupy a transverse disk of radius $5 \mathrm{fm}$ at $t=0$ and are formed at longitudinal proper time $\tau_{0}=0.1 \mathrm{fm}$ (i.e., the formation time for a particular particle $i$ is $t_{i}=\tau_{0} \times \cosh \left(\eta_{i}\right)$, $\left.z_{i}=\tau_{0} \times \sinh \left(\eta_{i}\right)\right)$.

We will compare results in different frames of ordering the collisions for the following schemes of doing cascade:

(a) Two gluons collide when their closest approach distance in the two particle center of mass frame is smaller than $\sqrt{\frac{\sigma}{\pi}}$ ( $\sigma$ is the scattering cross section). The collision space point is chosen to be the midpoint of the two particles in the two-body center of mass frame at their closest distance, the collisions are ordered in a global frame, either the collider lab frame or the target frame (they differ by 6 unit of rapidity boost);

(b) differs from (a) in that the collision space point for a particle (not for a collision) is the position of the particle in the two-body center of mass frame. So, in the global frame, for a particular parton parton collision, each parton will have its own time of scattering. The collisions are ordered according to the average time of the two scattering times in the global frame;

(c) differs from (b) in that the collisions are ordered according to the earlier time of the two scattering times; 
(d) two partons collide when their closest distance is smaller than $\sqrt{\frac{\sigma}{\pi}}$ in the global frame. The collisions are ordered according to the collision time in the global frame.

Different schemes specify different parton collision frame and different collision ordering time.

The system propagates from one collision to the next in the global frame instead of from one time step to the next.

Partons are all on mass shell. Rutherford cross section regulated by a screening mass, $\frac{d \sigma}{d t}=\frac{9 \pi \alpha_{S}^{2}}{2\left(\hat{t}-\mu^{2}\right)^{2}}$, is taken to generate the scattering angular distribution. In the above formula, $t=\left(p_{1}-p_{2}\right)^{2}$ in which $p_{1}$ and $p_{2}$ are 4 momenta of one particle before and after the collision. The total cross section for parton collision is taken as:

$$
\sigma=\frac{9 \pi \alpha_{S}^{2}}{2 \mu^{2}} .
$$

We set $\alpha_{S}=\sqrt{\frac{2}{9}} \approx 0.47$

Numerically [4], we divide the space into cells. We store only the next collision time, partner and next particle in the same cell in an interaction list to save memory and memory manipulation. The interaction list is only updated locally, i.e., only the cell where the collision happens and its neighboring cells are updated. This reduces the number of checks and significantly increases the speed of the parton cascade code. We can get one 6,000 collisions event in around 10 minutes. Our results have been checked by taking away the cells to make sure they are consistent with the ordinary calculations without space divisions. At the present stage, there are only 2 to 2 scatterings. We are working on including the radiation processes into the GPC.

\section{RESULTS FOR DIFFERENT SCHEMES}

The $\frac{d N}{d y}, \frac{d E_{T}}{d y}$ and $\frac{d N}{d p_{T}}$ distribution of partons are closely related to the hadronic observables. In this section, we are going to study parton cascade induced changes of these distributions. First, we look at the case when the mean free path is on the order of the interaction range $\sqrt{\frac{\sigma}{\pi}}$; then the case when the mean free path is much smaller. Further, we study the dependence of the results on the differential cross section, i.e., the angular distribution of outgoing particles from a collision. Finally, a discussion on the number of total collisions vs. the number of non-causal collisions and effects of a small particle mass.

The mean free path $l \sim \frac{1}{n \sigma}$ in which $n$ is the parton number density and the cross section $\sigma \sim \frac{\pi}{\mu^{2}}$. The density $n$ can be estimated through:

$$
n=\frac{1}{\pi R^{2} t} \frac{d N}{d y} .
$$

In our case, $R=5 \mathrm{fm}, \frac{d N}{d y}=400$ and $t \sim 0.2 \mathrm{fm}$ taken into account the spread of rapidity around the space time rapidity. We get $n \sim 25 / \mathrm{fm}^{3}$.

The interaction range $\sqrt{\frac{\sigma}{\pi}} \sim \frac{1}{\mu}$.

To have $l>\sqrt{\frac{\sigma}{\pi}}$, we need to have $\mu>4.3 \mathrm{fm}^{-1} \approx$ $0.85 \mathrm{GeV}$. This is a condition for isolated 2 body collisions that is generally violated. Ideally we also need many particles within the Debye screening scale:

$$
N_{D}=n\left(\frac{4}{3} \frac{\pi}{\mu^{3}}\right) \gg 1 .
$$

For $\mu=3 \mathrm{fm}^{-1}, N_{D} \approx \frac{100}{3^{3}} \approx 4$.

We showed below even though the isolated 2 body collision condition is barely satisfied, in the situation with strong inside-outside correlations like the system evolving from the initial conditions we specified above, a sensible scheme can still produce reasonable results.

Fig. 1, Fig. 2 and Fig. 3 give the $\frac{d N}{d y}, \frac{d E_{T}}{d y}$ and $\frac{d N}{d p_{T}}$ distributions with cross section $\sigma=\frac{\pi}{9} \mathrm{fm}^{2}(\alpha=0.47$ and $\mu=3 \mathrm{fm}^{-1}$ ) respectively. In plotting the target frame ordering case, we make a shift so that the central rapidity always has rapidity 0 .

After evolution, $\frac{d N}{d y}$ is almost the same as the initial. $\frac{d E_{T}}{d y}$ has a drop from initial value $\sim 385 \mathrm{GeV}$ to around $335 \mathrm{GeV}$. This shows the longitudinal work [5] done by the effective pressure. The absolute value of slope of $\frac{d N}{d p_{T}}$ log curve is increasing which clearly shows the effective cooling of the system. The results do not depend on the frame we use to order the collisions.

Scheme (b) and (c) give similar results.

A close look at the average number of collisions per event shows that for scheme (a) we get $\sim 6900$ collisions, for (b) 6800 and for (c) 6200 when collision order is in the collider lab frame. In the target frame case, each gets several hundred less.

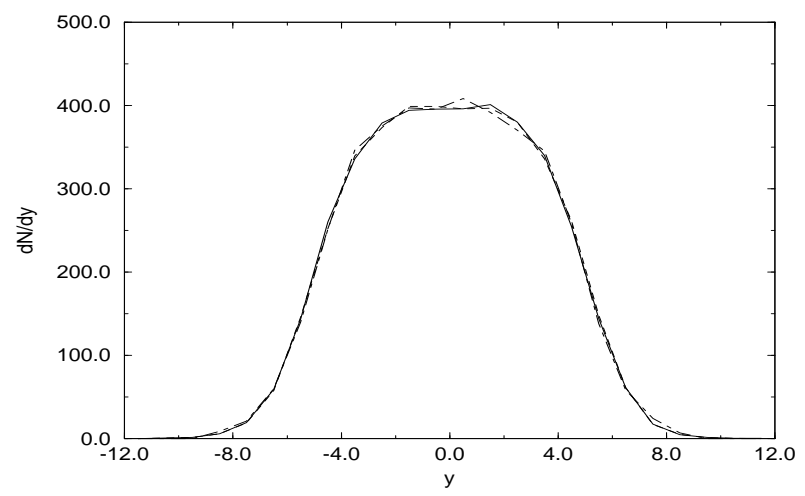


FIG. 1. 20 event averaged $\frac{d N}{d y}$ distribution for scheme (a) with $\alpha=0.47$ and $\mu=3 \mathrm{fm}^{-1}$. The dot-dashed curve is the initial distribution for reference; the solid curve is for the case that collisions are ordered in the collider center of mass frame; for the dashed curve, the collisions are ordered in the target frame.

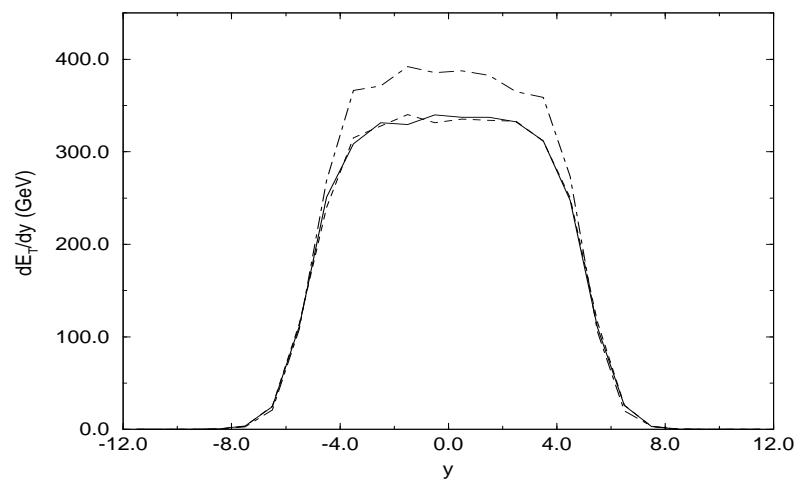

FIG. 2. 20 event averaged $\frac{d E_{T}}{d y}$ distribution for scheme (a) with $\alpha=0.47$ and $\mu=3 \mathrm{fm}^{-1}$. The dot-dashed, solid and dashed curves are for the initial, collider frame ordering and target frame ordering respectively.

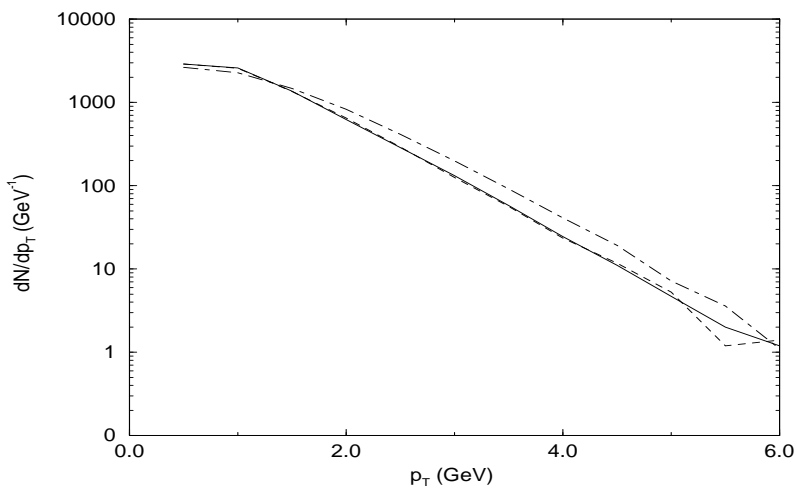

FIG. 3. 20 event averaged $\frac{d N}{d p_{T}}$ distribution for scheme (a) with $\alpha=0.47$ and $\mu=3 \mathrm{fm}^{-1}$. The dot-dashed, solid and dashed curves are for the initial, collider frame ordering and target frame ordering respectively.

Fig. 4, Fig. 5 and Fig. 6 give the results for scheme (d). Strong frame dependence is observed in this case. From Fig. 4 and 5 , we see that a boost of the system by 6 unit of rapidity (the original 0 rapidity goes to -6 before shifting back to 0) makes a peak which is shifted from the central along the boost by 2 unit of rapidity. We use a much smaller $\sigma=\frac{\pi}{25} \mathrm{fm}^{2} \approx 1 \mathrm{mb}$ cross section because in scheme (d), we get much more collisions even with a smaller cross section. In the collider frame ordering case, there are around 22,000 collisions per event; in the target frame ordering case, we get 97,000 !

The frame dependence of scheme (d) is due to the rapidity correlation introduced by taking the closest distance in the global frame. It neglects the flow velocity and increases the density of the particles. This is also seen in the huge increase of number of collisions with smaller cross section comparing to the other schemes. Scheme (a), (b) and (c) take into account of the flow by looking at the closest distance in the 2 particle center of mass frame. This corresponds to the time in the 2 particle center of mass frame when the 2 discs meet.

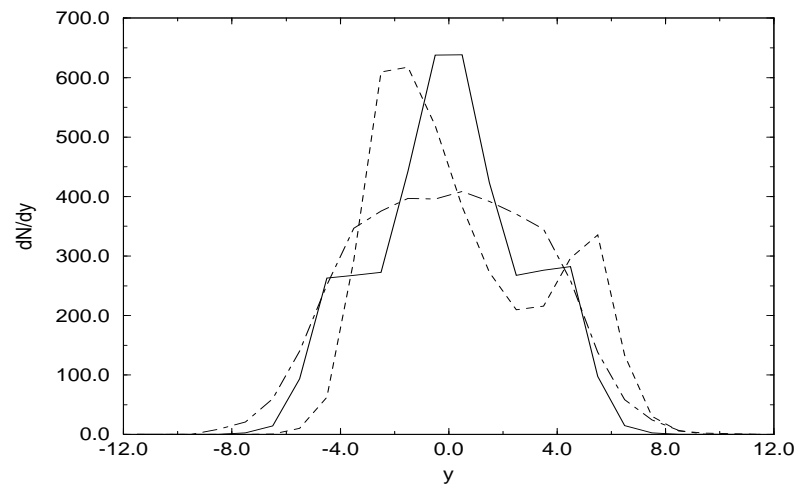

FIG. 4. 5 event averaged $\frac{d N}{d y}$ distribution for scheme (d) with $\alpha=0.47$ and $\mu=5 \mathrm{fm}^{-1}$. The dot-dashed, solid and dashed curves are for the initial, collider frame ordering and target frame ordering respectively.

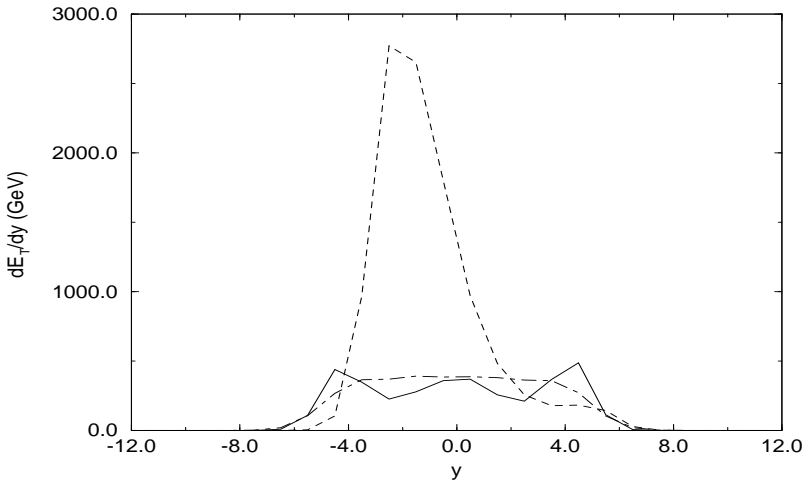

FIG. 5. 5 event averaged $\frac{d E_{T}}{d y}$ distribution for scheme (d) with $\alpha=0.47$ and $\mu=5 \mathrm{fm}^{-1}$. The dot-dashed, solid and dashed curves are for the initial, collider frame ordering and target frame ordering respectively. 


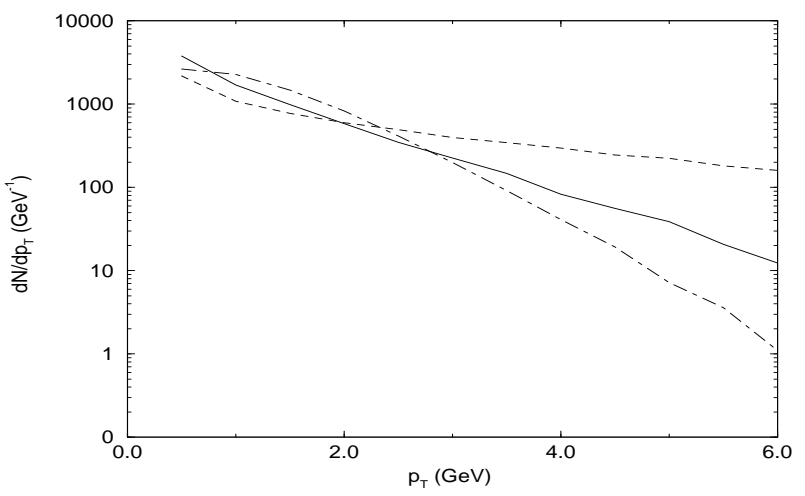

FIG. 6. 5 event averaged $\frac{d N}{d p_{T}}$ distribution for scheme (d) with $\alpha=0.47$ and $\mu=5 \mathrm{fm}^{-1}$. The dot-dashed, solid and dashed curves are for the initial, collider frame ordering and target frame ordering respectively.

Fig. 7, Fig. 8 and Fig. 9 give results for $\sigma=\pi \mathrm{fm}^{2}$. Now the interaction range is $1 \mathrm{fm}$ and the mean free path is initially $\frac{1}{25 \pi} \sim 0.01 \mathrm{fm}$. For $\alpha=0.47$ and $\mu=1 \mathrm{fm}^{-1}$, the $d N / d y$ distribution has a dip in the middle for the collider frame ordering case, while the target frame ordering case doesn't have one. The $d E_{T} / d y$ central rapidity plateau height is almost the same as the for $\alpha=0.47$ and $\mu=3 \mathrm{fm}^{-1}$ case. This is because of the effective screening mass is used to regulate the forward scattering cross section and larger cross section or more forward collisions does not lead to more collective work. This is clearly shown when we vary the differential cross section by change the $\alpha$ from 0.47 to 1.41 and $\mu$ from $1 \mathrm{fm}^{-1}$ to $3 \mathrm{fm}^{-1}$. As shown in Fig. 8, the collider frame ordering case has a clear drop at rapidity from -4 to -2 and from 2 to 4 . In the middle, there is a peak. The target frame ordering has a lower plateau. The $d E_{T} / d y$ is frame dependent now. The $d N / d p_{T}$ for $\alpha=1.41$ and $\mu=3 \mathrm{fm}^{-1}$ has a lower temperature parameter than that of $\alpha=0.47$ and $\mu=1 \mathrm{fm}^{-1}$. This is because more work is been done when there are more relatively large angle scatterings and we get more cooling. Scheme (b) and (c) can not get frame independent results either.

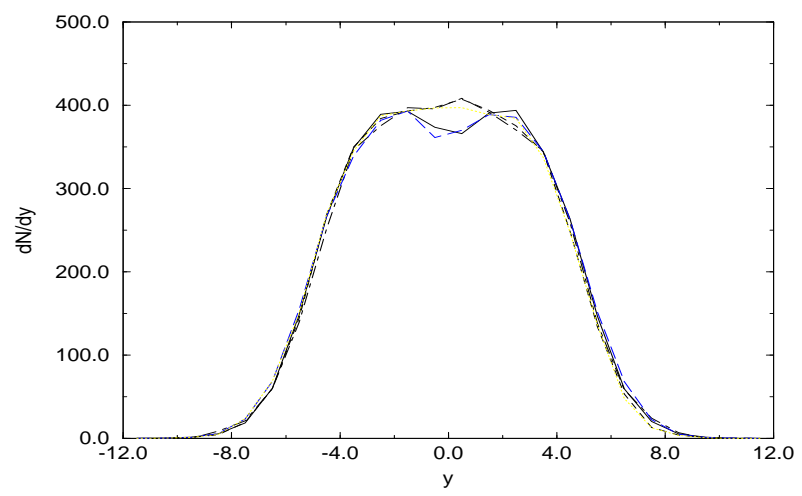

FIG. 7. 20 event averaged $\frac{d N}{d y}$ distribution for scheme (a). The dot-dashed curve is for the initial distribution; the thick solid is for $\alpha=0.47$ and $\mu=1 \mathrm{fm}^{-1}$ collider frame ordering; the thick dashed curve is for $\alpha=0.47$ and $\mu=1 \mathrm{fm}^{-1}$ target frame ordering; the long dashed curve is for $\alpha=1.41$ and $\mu=3 \mathrm{fm}^{-1}$ collider frame ordering; the dotted curve is for $\alpha=1.41$ and $\mu=3 \mathrm{fm}^{-1}$ target frame ordering. The dot-dashed, thick dashed and dotted curves overlap; the thick solid and long dashed curves overlap.

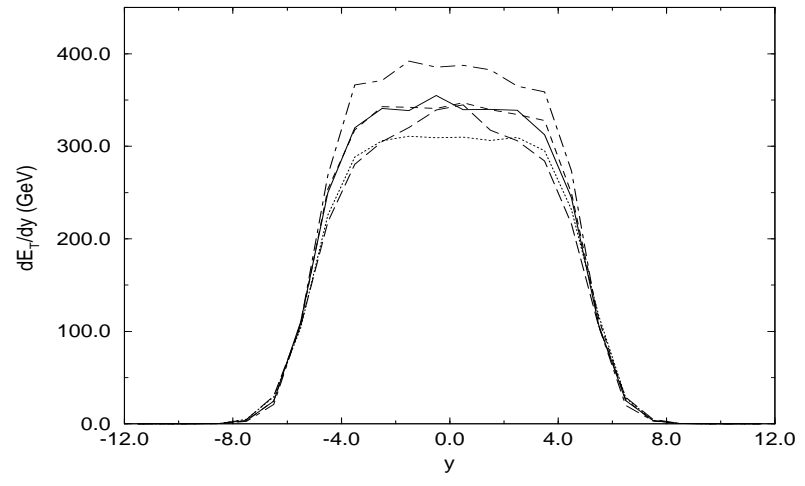

FIG. 8. 20 event averaged $\frac{d E_{T}}{d y}$ distribution for scheme (a). The dot-dashed curve is for the initial distribution; the thick solid is for $\alpha=0.47$ and $\mu=1 \mathrm{fm}^{-1}$ collider frame ordering; the thick dashed curve is for $\alpha=0.47$ and $\mu=1 \mathrm{fm}^{-1}$ target frame ordering; the long dashed curve is for $\alpha=1.41$ and $\mu=3 \mathrm{fm}^{-1}$ collider frame ordering; the dotted curve is for $\alpha=1.41$ and $\mu=3 \mathrm{fm}^{-1}$ target frame ordering.

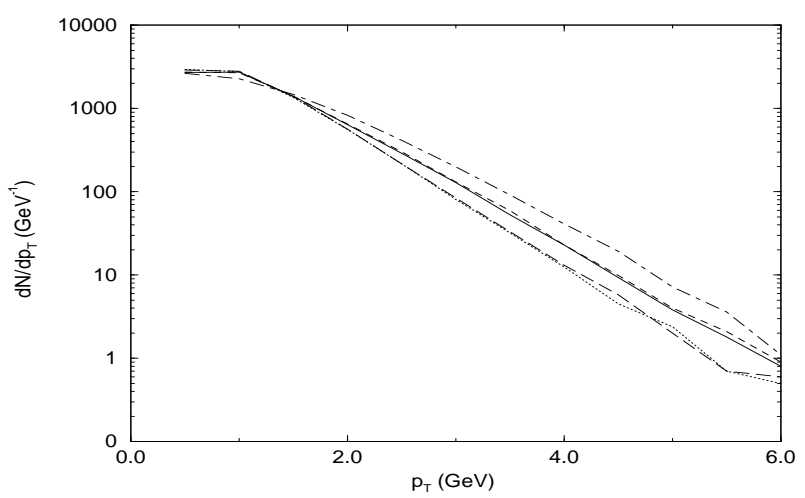

FIG. 9. 20 event averaged $\frac{d N}{d p_{T}}$ distribution for scheme (a). The dot-dashed curve is for the initial distribution; the thick solid is for $\alpha=0.47$ and $\mu=1 \mathrm{fm}^{-1}$ collider frame ordering; the thick dashed curve is for $\alpha=0.47$ and $\mu=1 \mathrm{fm}^{-1}$ target frame ordering; the long dashed curve is for $\alpha=1.41$ and $\mu=3 \mathrm{fm}^{-1}$ collider frame ordering; the dotted curve is for $\alpha=1.41$ and $\mu=3 \mathrm{fm}^{-1}$ target frame ordering. 
To study the effect of different differential cross sections, we compare the results for $\alpha=0.47$ and $\mu=$ $3 \mathrm{fm}^{-1}$ and $\alpha=1.41$ and $\mu=9 \mathrm{fm}^{-1}$. Shown in Fig. 10 and Fig. 11 are $d E_{T} / d y$ and $d N / d p_{T}$ distributions. $d N / d y$ distribution is the same for these two cases and they are the same as the initial. From Fig. 10, a clear drop in the height of central rapidity plateau can be seen with more large angle scatterings. This shows that more work has been done and is also the reason that we get more cooling in $d N / d p_{T}$ distribution. We get 7,000 collisions in the collider frame and 6,700 in the target frame. Scheme (b) gives the similar results and they are all frame independent.

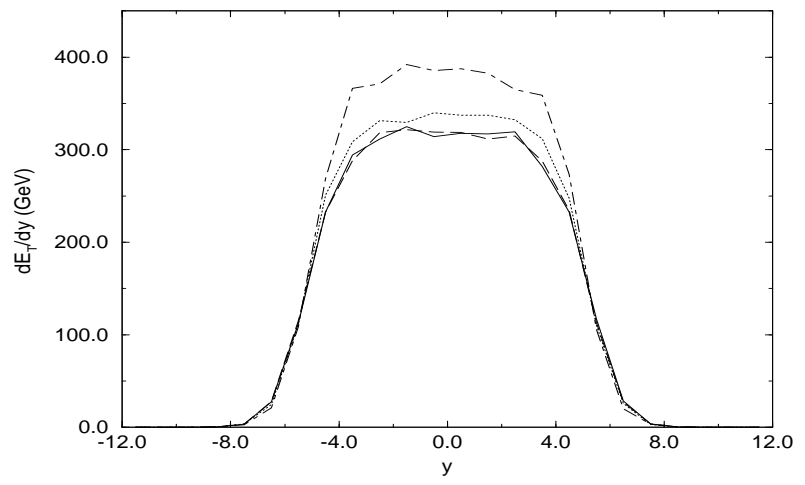

FIG. 10. 20 event averaged $\frac{d E_{T}}{d y}$ distribution for scheme (a). The dot-dashed curve is for the initial distribution; the dotted curve is for $\alpha=0.47$ and $\mu=3 \mathrm{fm}^{-1}$ the thick solid is for $\alpha=1.41$ and $\mu=9 \mathrm{fm}^{-1}$ collider frame ordering; the thick dashed curve is for $\alpha=1.41$ and $\mu=9 \mathrm{fm}^{-1}$ target frame ordering.

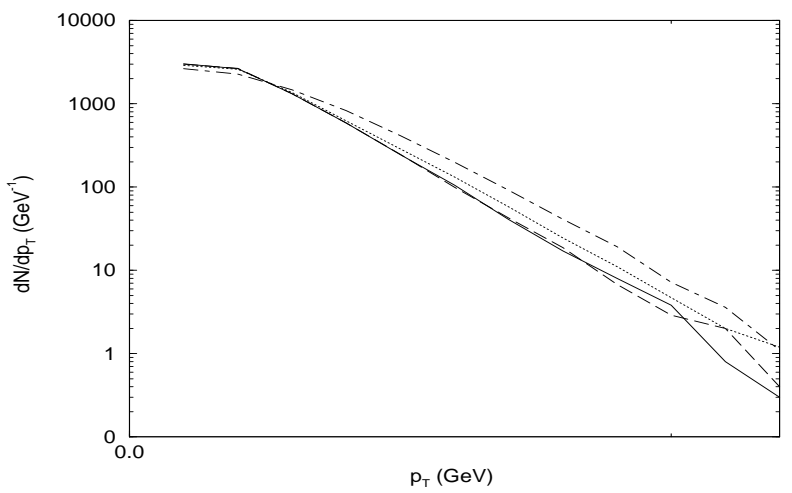

FIG. 11. 20 event averaged $\frac{d N}{d p_{T}}$ distribution for scheme (a). The dot-dashed curve is for the initial distribution; the dotted curve is for $\alpha=0.47$ and $\mu=3 \mathrm{fm}^{-1}$ the thick solid is for $\alpha=1.41$ and $\mu=9 \mathrm{fm}^{-1}$ collider frame ordering; the thick dashed curve is for $\alpha=1.41$ and $\mu=9 \mathrm{fm}^{-1}$ target frame ordering.
Another interesting thing to look at is the number of non-causal collisions vs. the number of total collisions. A non-causal collision here is defined to be a collision that the global ordering is not consistent with the ordering for a particular particle participating in the collision. This is better seen in scheme (b). If particle a's next collision according to global ordering is with $\mathrm{i}$, but the collision time for particle a with i (i.e., the time a is going to change its momentum) is later than the collision time for a with $\mathrm{j}$ (even though according to global ordering, a j collision happens later), then we call this collision a noncausal collision. For scheme (b) in the collider lab frame, with $\alpha=0.47$ and $\mu=3 \mathrm{fm}^{-1}$, we get a total of 6,800 collisions per event with 900 of them non-causal. With a larger cross section $\left(\alpha=0.47\right.$ and $\left.\mu=1 \mathrm{fm}^{-1}\right)$, as we expect, the non-causal to total ratio goes up. There are total 21,000 collisions with 10,400 of them non-causal. Boost to the target frame, the total number of collisions for $\alpha=0.47$ and $\mu=3 \mathrm{fm}^{-1}$ is 6,500 while the number of non-causal is 1,000. The effects of putting in a mass for the particles are also studied. There are no significant changes in $\frac{d E_{T}}{d y}, \frac{d N}{d y}$ and $\frac{d N}{d p_{T}}$ and the number of total and non-causal collisions for massless, $m=0.01 \mathrm{GeV}$ and $m=0.1 \mathrm{GeV}$. Fig. 12 shows the collisions rates. We see that the results are not sensitive to small masses of the particles.

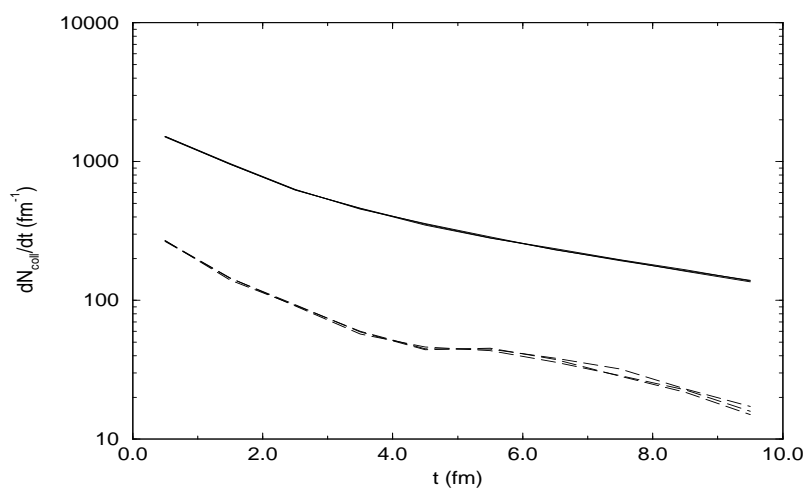

FIG. 12. 20 event averaged collision rate as a function of time for scheme (b). The upper 3 solid curves are for massless, $m=0.01 G e V$ and $m=0.1 G e V$ total collision rate and the lower 3 dashed curves are for non-causal collision rate of these 3 masses.

What we describe here is the parallel ensemble method in which average is taken over $n$ independent events. An alternative, the full ensemble method [6], is to increase the particle number by $k$ (by dividing the particles into small pieces) and at the same time decrease the cross section by a factor of $k$. In the full ensemble method, the mean free path is the same as for the parallel ensemble method, but the cross section is smaller and hence isolated 2 body scattering condition can be satisfied. This 
makes it possible to eliminate the causality violation in the limit of large $k$. But the full ensemble method will increase the computation time significantly due to the increasing number of checks required for the higher density system. We see from the above study that for reactions with strong inside outside correlations, the full ensemble method may not have to be used in practice to get reasonable results as long as a sensible scattering prescription is used.

\section{CONCLUSIONS}

After studying the frame dependence of parton cascade results, we see that different schemes may give quite different results. Especially, the global frame collision and ordering scheme results have a strong frame dependence. Other 2 particle center of mass frame collision and global frame ordering schemes can give almost frame independent results even when the interaction range is on the order of the mean free path. As we expect, they fail when the interaction is much large than the mean free path. Different differential cross sections lead to different longitudinal work and give different $d E_{T} / d y$ and $d N / d p_{T}$ results. Since radiation will increase the cross section and parton density, the quantum statistics will also change effective cross sections, further study is necessary when we apply this to more realistic situations.

\section{ACKNOWLEDGMENTS}

We thank M. Asakawa, S. Gavin, K. Geiger, E. Gonzalez-Ferreiro, D. Kahana, S. Kahana, Z. Lin, R. Mattiello, C. Noack, D. Rischke, J. Randrup, R. Vogt, K. Werner for useful discussions, and M. Gyulassy for continuing encouragement and discussions and a critical reading of the manuscript. B. Z. gratefully acknowledges partial support from the [Department of Energy] Institute for Nuclear Theory at the University of Washington program INT-96-3 during the completion of this work. This work was supported by the U.S. Department of Energy under Contract No. DE-FG02-93ER40764 and DEFG-02-92ER40699.

[1] Y. Pang, D. E. Kahana, S. H. Kahana and T. J. Schlagel, Nucl. Phys. A 590 (1995) 565c;

H. Sorge, H. Stöker and W. Greiner, Nucl. Phys. A 498 (1989) 567c; K. Geiger, Phys. Rev. D 46 (1992) 4965, 4986.

[2] e.g., T. Kodama, S. B. Duarte, K. C. Chung, R. Donangelo and R. A. M. S. Nazareth, Phys. Rev. C 29 (1984) 2146;
G. Peter, D. Behrens and C. C. Noack, Phys. Rev. C 49 (1994) 3253.

[3] e.g. see G. Kortmeyer, W. Bauer, K. Haglin, J. Murray and S. Pratt, it Phys. Rev. C 522714.

[4] Further details will be published later.

[5] P. V. Ruuskannen, Phys. Lett. 147 B (1984) 465; K. J. Eskola and M. Gyulassy, Phys. Rev. C 47 (1993) 2329 ;

B. Zhang, M. Gyulassy and Y. Pang, CU-TP-795.

[6] see e.g., G. Welke, R. Malflied, C. Gregoire, M. Prakash and E. Suraud, Phys. rev. C 40 (1989) 2611; Kortemeyer et.al. cited above. 